\title{
Z Research Square \\ Synergetic Modulation of Plant Cadmium Tolerance via MYB75-mediated ROS Homeostasis and Transcriptional Regulation
}

\section{Ting Zheng}

Sichuan Normal University https://orcid.org/0000-0003-1347-9404

\section{Feng Yang}

Sichuan University

Dawei Zhang ( $\nabla$ zhdawei@scu.edu.cn )

Sichuan University

\section{Research Article}

Keywords: Cadmium (Cd), Synergetic modulation, MYB75-mediated, ROS homeostasis, transcriptional regulation

Posted Date: January 20th, 2022

DOI: https://doi.org/10.21203/rs.3.rs-1245108/v1

License: (c) (i) This work is licensed under a Creative Commons Attribution 4.0 International License. Read Full License 


\section{Abstract}

Cadmium (Cd) is a heavy metal with biological toxicity, which can be detoxified through chelation and compartmentation in plants. Transcriptional regulation mediates plant $\mathrm{Cd}$ tolerance by modulating these processes. However, the mechanism remains to be studied. Our results showed that a previously unknown function of MYB75 transcription factor in the regulation of Cd tolerance. Cd exposure stimulates anthocyanin accumulation by raising MYB75 expression. Enhanced Cd tolerance was observed in the MYB75-overexpressing plants, whereas increased Cd sensitivity was found in the MYB75 loss-of-function mutants. Under Cd stress conditions, lower reactive oxygen species (ROS) levels were detected in MYB75-overexpressing plants than in wild type plants. In contrast, higher ROS levels were found in MYB75 loss-of-function mutants. Overexpression of MYB75 were associated with increased glutathione (GSH) and phytochelatin (PC) content by anthocyanin-mediated ROS homeostasis. Furthermore, the expression of Cd stress-related gene including $A C B P 2$ and $A B C C 2$ was elevated in MYB75-overexpressing plants, and this upregulation was mediated through the mechanism by which MYB75 directly bind to the promoter of $A C B P 2$ and $A B C C 2$. Our findings reveal an important role for MYB75 in regulation of plant Cd tolerance via anthocyanin-mediated ROS homeostasis and PC content, and through upregulation of $\mathrm{Cd}$ stress-related gene at transcriptional level.

\section{Key Message}

MYB75 enhances plant cadmium tolerance by mediating ROS homeostasis and cadmium tolerancerelated genes expression.

\section{Introduction}

Human production processes such as industry and mining can increase concentrations of heavy metals in soil, air and water. The accumulation of heavy metals in soil can lead to the loss of soil fertility, the reduction of vegetation, and the pollution of water sources (Clemens et al., 2016, Zou et al., 2021). Cadmium (Cd) is a widespread heavy metal contamination, which has a serious toxic effect on plants, animals and human (Clemens, 2019). Cd is a nonessential and toxic element for plant growth and development. It is commonly known that $\mathrm{Cd}$ in water can rapidly enter plant via root, resulting in toxicological symptoms (Haider et al., 2021). Firstly, Cd can bind to the sulfhydryl, histidine, and carboxyl of structural protein, thus inhibiting protein function (Huybrechts et al., 2019). Cd also has similar chemical properties to other divalent cation, which hence can replace the metal ion in structural protein and cause impairment of protein function (Cuypers et al., 2010). Further, although Cd has non-redox property, it can indirectly induce the production of reactive oxygen species (ROS) (Wu et al., 2019, Zhang et al., 2020). ROS-mediated change of redox environment in plant cell can disrupt protein function and destroy cell structure, thus inhibiting physiological metabolic processes such as photosynthesis, respiration, photorespiration, and cell cycle (Waszczak et al., 2018). Consequently, improvement of Cd tolerance is critical for plant growth and development. 
Cd in agricultural soil is mainly derived from atmospheric deposition and industrial effluent. Cd accumulation in plant is mostly due to the uptake of soil $\mathrm{Cd}$ via root. Previous researches have established that various plant transporters play an important role in uptake of $\mathrm{Cd}$. Cd uptake and transport was implicated in several genes encoding transporters including OsNramp5, HMA2, HMA4, IRT1, and PDR8 (Chang et al., 2020, Sheng et al., 2019, Wong et al., 2009a, Wong et al., 2009b, Wu et al., 2021). Cd is transported into and accumulated in plants, which is harmful to plant growth and development. But plants have evolved multiple defense mechanisms to resist and reduce the toxicity of Cd. Initially, $\mathrm{Cd}^{2+}$ chelated by Glutathione (GSH) and phytochelatin (PC) can sequester into vacuoles (González et al., 2021). Some transcription factors including ZAT6 and WRKY12, regulate Cd tolerance via modulation of GSH and PC biosynthesis at transcriptional level (Chen et al., 2016, Han et al., 2019). Next, plants may mediate the expression of metal uptake transporter genes and efflux transporter genes to compartment Cd distribution (Sheng et al., 2019). Finally, some membrane protein proteins such as ACBP2 and FP6 can bind $\mathrm{Cd}^{2+}$ via sulfhydryl groups on cysteine residues to form nontoxic or less toxic compound (Gao et al., 2010, Gao et al., 2009). PDF2.5 promotes Cd transfer from protoplast to cell wall, thus alleviating $\mathrm{Cd}$ toxicity (Luo et al., 2019). In addition, to decrease Cd-induced ROS accumulation, plants enhance ROS scavenging by activating antioxidant system. Plant Cd tolerance was enhanced by mediating these collective mechanisms at multiple levels, especially at transcriptional level.

MYB family transcription factors play a significant role in plant developmental processes as well as plant tolerance to diverse environmental stresses. Data from several studies suggest that MYB transcription factors participate in regulation of plant $\mathrm{Cd}$ tolerance, but details of the regulation mechanism need further research. MYB75, which is also defined as PRODUCTION OF ANTHOCYANIN PIGMENT 1 (PAP1), plays a key role in anthocyanin accumulation (Borevitz et al., 2000, He et al., 2021). However, the possible role of MYB75 in Cd tolerance and the details of the underlying mechanism has not been characterized.

To enrich the regulatory mechanisms in under Cd exposure, here we identify MYB75 as a new regulator of plant $\mathrm{Cd}$ tolerance. Under $\mathrm{Cd}$ stress, a myb75-c mutant shows increased sensitivity to $\mathrm{Cd}$ stress and accumulates more ROS but less PC, whereas the transgenic lines overexpressing MYB75 decrease ROS levels and lead to elevate PC content compared with wild type plants. Furthermore, MYB75 directly binds to the promoter of $\mathrm{Cd}$ stress-related gene including $A C B P 2$ and $A B C C 2$, thus raising their transcription. Our study exposes that MYB75 acts as a positive regulator of $\mathrm{Cd}$ tolerance by anthocyanin-mediated ROS homeostasis and PC content, and through targeting $A C B P 2$ and $A B C C 2$ in Arabidopsis.

\section{Materials And Methods}

\section{Plant materials and growth conditions}

The Arabidopsis thaliana pap 1-D, 35S: MYB75 and myb75-c were described as previously (Zheng et al., 2020a, Zheng et al., 2019). The $1 / 2 \mathrm{MS}$ with Arabidopsis seeds were placed at $4{ }^{\circ} \mathrm{C}$ for $2 \mathrm{~d}$ before moving to $22{ }^{\circ} \mathrm{C}$ under diverse light conditions. For $\mathrm{Cd}$ treatment, $100 \mathrm{mM} \mathrm{CdCl}_{2}$ was added to the $1 / 2 \mathrm{MS}$ medium ( $\mathrm{pH}$ 5.8) to a final concentration of $50 \mu \mathrm{M} \mathrm{CdCl}_{2}$, and $75 \mu \mathrm{M} \mathrm{CdCl}_{2}$. Three-day-old wild type and 
mutant or transgenic seedlings were transferred to $1 / 2 \mathrm{MS}$ agar plates in the absence or presence of $\mathrm{CdCl}_{2}$ for the indicated number of days. To reduce variation because of the precipitation of heavy metals, wild type and mutant or transgenic plants were grown adjacent to each other in the same plate and their growth was compared. After the indicated days of growth, the plants were sampled for root growth and fresh weight assays. Nicotiana benthamiana grown in soil at $22{ }^{\circ} \mathrm{C}$ under 16 -h-light/8-h-dark conditions was used for the transient expression assays.

\section{Chlorophyll measurement}

The determination of chlorophyll contents was measured as described (Zhang et al., 2021). Briefly, a total of $100 \mathrm{mg}$ of plant materials was pulverized with liquid nitrogen, incubated in a $1.0 \mathrm{~mL}$ of $80 \%$ acetone in the dark for $30 \mathrm{~min}$ and centrifuged for $5 \mathrm{~min}$ at $12000 \mathrm{rpm}$. Absorbance of the supernatant was measured at 645 and $663 \mathrm{~nm}$ and then total chlorophyll content was calculated.

\section{Analysis of chlorophyll fluorescence}

Chlorophyll fluorescence was determined with an pulse-modulated fluorometer (FMS-2, Hansatech, UK). For measurement of $F_{v} / F_{m}$, plants were dark adapted for $30 \mathrm{~min}$. Minimal fluorescence $\left(F_{0}\right)$ was measured during the weak measuring pulses, and maximal fluorescence $\left(F_{m}\right)$ was measured by $0.8 \mathrm{~s}$ pulse of light at about $4,000 \mathrm{mmol} \mathrm{m}^{-2} \mathrm{~s}^{-1}$. An actinic light source was then applied to obtain steadystate fluorescence yield $\left(F_{s}\right)$, after which a second saturation pulse was applied for $0.7 \mathrm{~s}$ to obtain lightadapted maximum fluorescence $\left(F_{m}\right) . F_{v} / F_{m}$, ФPSIl were calculated as $F_{m}-F_{0} / F_{m}\left(F_{m}{ }^{\prime} F_{s}\right) / F_{m}{ }^{\prime}$ ' respectively (Baker, 2008).

\section{Ion leakage assay}

The determination of ion leakage was measured as described (Ding et al., 2018). Summarily, the injury seedlings were put into $15 \mathrm{ml}$ tubes containing $5 \mathrm{ml}$ deionized water, which were shaken at $22^{\circ} \mathrm{C}$ for 15 min, and the conductivity was measured as $\mathrm{S} 1$. After detecting $\mathrm{S} 1$, the tubes were put into boiled water at $100{ }^{\circ} \mathrm{C}$ for $15 \mathrm{~min}$ and shaken at $22^{\circ} \mathrm{C}$ for $1 \mathrm{~h}$, and then, S2 was measured. The formula (S1-S0)/(S2-S0) was used to calculate ion leakage (S0: conductivity of deionized water).

\section{Oxidative damage estimation, in situ superoxide and $\mathrm{H}_{2} \mathrm{O}_{2}$ staining}

Superoxide and $\mathrm{H}_{2} \mathrm{O}_{2}$ levels were visually detected with nitro blue tetrazolium (NBT) and 3, 3diaminobenzidine (DAB), respectively, as described previously (Zou et al., 2016). Seedlings were excised at the base with a razorblade and supplied through the cut ends with NBT $\left(0.5 \mathrm{mg} \mathrm{ml}^{-1}\right)$ or DAB $(2 \mathrm{mg}$ $\mathrm{ml}^{-1}$ ) solutions for $8 \mathrm{~h}$. Leaves were then decolorized in boiling ethanol (95\%) for $20 \mathrm{~min}$. At least three seedlings were used for each treatment.

$\mathrm{O}_{2}{ }^{--}$and $\mathrm{H}_{2} \mathrm{O}_{2}$ content was measured as described (Lei et al., 2020). Briefly, the $\mathrm{O}_{2}{ }^{--}$reacts with MSDS (hydroxylamine hydrochloride) to generate $\mathrm{NO}^{2-}$. Then a red azo compound is generated with the reaction 
of p-aminobenzenesulfonic acid and a-naphthylamine, which possesses an absorption peak at $530 \mathrm{~nm}$. The yellow titanium peroxide composite generated by the reaction of $\mathrm{H}_{2} \mathrm{O}_{2}$ which has an absorption peak at $415 \mathrm{~nm}$ is used to calculate the $\mathrm{H}_{2} \mathrm{O}_{2}$ content. Each sample has three biological repeats.

\section{Determination of antioxidant enzymes}

For the enzyme assays, $0.3 \mathrm{~g}$ of leaf were ground with $3 \mathrm{ml}$ ice cold $25 \mathrm{mM}$ Hepes buffer ( $\mathrm{pH} 7.8$ ) containing $0.2 \mathrm{mM}$ EDTA, $2 \mathrm{mM}$ ascorbate and $2 \%$ polyvinylpyrrolidone (PVP) (Zou et al., 2016). The homogenates were centrifuged at $4{ }^{\circ} \mathrm{C}$ for $20 \mathrm{~min}$ at 12,000 $\mathrm{g}$ and the resulting supernatants were used for the determination of enzymatic activity. Superoxide dismutase (SOD), catalase (CAT), ascorbate peroxidase (APX), peroxidase (POD) activities were assayed as described previously.

\section{Real-time PCR analysis}

RNA was extracted as described previously, and cDNA synthesis was performed by one microgram (Zhang et al., 2010). qRT-PCR analysis was conducted by using SYBR Green PCR Master Mix. Three separate experiments and technical triplicates of each experiment were implemented. Gene expression was standardized to the ACTIN 8 transcript levels.

\section{Cloning and transient expression assay}

The 2000 bp promoters of $A C B P 2$ and $A B C C 2$ were PCR-amplified from Arabidopsis genomic DNA using specific primers (Supplementary Table S1). These promoters were cloned into the vector YY45 (YFP substitutes for $\beta$-glucuronidase [GUS] reporter), named PrOACBP2: GUS and ProABCC2: GUS. These two constructs were co-transformed into epidermal cells of Nicotiana benthamiana with the 35S: MYB75-HA construct. Samples were then collected and labelled, and GUS staining was performed as described previously.

\section{LUC reporter transactivation assays}

The 2000 bp promoters of ACBP2 and ABCC2 were PCR-amplified from Arabidopsis genomic DNA using specific primers (Supplementary Table S1). These promoters were cloned into the vector pGreenll0800LUC (Li et al., 2016), named ProACBP2: LUC and ProABCC2: LUC. These two constructs were cotransformed into wild type protoplasts with the 35S: MYB75-HA construct. For LUC assay, $100 \mu \mathrm{l}$ of protoplast lysis buffer was added to the frozen protoplasts and mixed with a pipette gun (Yoo et al., 2007). After $5 \mathrm{~min}$ incubation on ice, $20 \mathrm{ml}$ of the lysate harvested by centrifugation at $1,000 \mathrm{~g}$ for $2 \mathrm{~min}$ and $100 \mathrm{ml}$ LUC mix were used to measure LUC activity.

\section{Electrophoresis Mobility Shift Assay}

EMSAs were performed as previously described (Zhang et al., 2021). Briefly, MBP-MYB75 was expressed in E. coli and purified using MBP Sepharose beads, respectively. The DNA fragment (Supplementary Table S1) was incubated with MBP-MYB75 in EMSA binding buffer (25 mM HEPES-KOH, pH 8.0, $50 \mathrm{mM} \mathrm{KCl,} 1$ $\mathrm{mM}$ dithiothreitol [DTT] and 10\% glycerol). An Electrophoretic Mobility Shift Assay (EMSA) Kit with SYBR Green and SYPRO Ruby EMSA stains (Thermo Fisher) were used to detect protein-DNA interactions. 


\section{ChIP assays}

ChIP assays perform as described (Yu et al., 2008). The 4-week-old plants collected in $50 \mathrm{~mL}$ tubes, and $37 \mathrm{~mL} \mathrm{1 \%}$ formaldehyde solution was used for cross-linked under a vacuum for $20 \mathrm{~min}$. The chromatin was collected and sheared by sonication to reduce the average DNA fragment size to around $500 \mathrm{bps}$, then the sonicated chromatin complex was immunoprecipitated by specific antibodies anti-HA. After reverse cross-linking, the immunoprecipitated DNA fragment was analysed by qPCR with specific primers shown in Supplementary Table S1.

\section{Statistical analysis}

Samples were analyzed in three individual biological replicates, and the data are indicated as the mean \pm SD. Two-way ANOVA (LSD's multiple-range test) or Student's t-test were performed at a significance level of $\mathrm{P}<0.05$.

\section{Accession numbers}

The Arabidopsis Genome Initiative identifiers for the genes described in this article are as follows: MYB75 (AT1G56650), MYB90 (AT1G66390), TT8 (AT4G09820), EGL3 (AT1G63650), TTG1 (AT5G24520), ACBP2 (AT4G27780), ABCC2 (AT2G34660), GSH1 (AT4G23100), PDR8 (AT1G59870), ATM3 (AT5G58270), PDF2.5 (AT5G63660) and ACTIN 8 (AT1G49240).

\section{Results}

\section{MYB75 transcription factor participates in modulation of plant cadmium tolerance}

Previous research has proved that plants exposed to Cd stress show transcriptional change of genes involved in phenylpropanoid metabolisms and anthocyanin accumulation (Dai et al., 2012, Herbette et al., 2006), thus we further investigated the mechanism underlying Cd stress-regulated anthocyanin accumulation. To test the impact of $\mathrm{Cd}$ stress on anthocyanin accumulation, seeds of the wild type were germinated on one-half-strength Murashige and Skoog (1/2 MS) agar plates containing either $0,25,50$, $75,100 \mu \mathrm{M} \mathrm{CdCl}_{2}$ for $3 \mathrm{~d}$. As shown in Fig. $1 \mathrm{~A}$, anthocyanin accumulation was elevated in wild type under $\mathrm{Cd}$ stress. Quantification of anthocyanin also verified that the anthocyanin level increased with the $\mathrm{Cd}$ concentration (Fig. 1B). Previous evidence demonstrated that anthocyanin biosynthesis derives from flavonoid biosynthetic pathway and three anthocyanin-specific genes encoding dihydroflavonol 4reductasae (DFR), leucoanthocyanidin dioxygenase (LDOX), UDP-glucose: flavonoid 3-oglucosyl transferase (UF3GT) have been identified, and expression of these genes is regulated by MYB-bHLHWD40 (MWB) protein complex (Xu et al., 2015). We hence determined the expression of regulatory gene in wild type without or with $25,50,75,100 \mu \mathrm{M} \mathrm{CdCl}_{2} . \mathrm{MYB} 75$ transcription was notably induced in response to Cd stress (Fig. 1B, Fig. S1A). However, Cd treatment would not alter the expression of other regulatory 
gene (MYB90, TT8 and EGL3) (Fig. S1B-D). These results suggest that MYB75 participates in Cd stressinduced anthocyanin accumulation.

Above findings lead us conclude that MYB75 transcription factor is involved in modulation of plant Cd tolerance. Consequently, pap1-D seedlings, the activation tag mutant constitutively overexpresses MYB75/PAP1 and myb75-c that MYB75 knockout mutant using the CRISPR-Cas9 system were used for determining the MYB75 function in plant Cd tolerance. Arabidopsis seedlings grown vertically in 1/2 MS agar plates without $\mathrm{CdCl}_{2}$ for $3 \mathrm{~d}$, then were moved to $1 / 2 \mathrm{MS}$ agar plates without or with 50 or $75 \mu \mathrm{M}$ $\mathrm{CdCl}_{2}$ for $7 \mathrm{~d}$. As can be seen from Fig. $1 \mathrm{C}$, when grown on 1/2 MS media without $\mathrm{CdCl}_{2}$, pap 1-D and myb75-c exhibited no difference compared with wild type. Nevertheless, pap 1-D showed higher tolerance in response to $\mathrm{Cd}$ stress compared with wild type (Fig. 1C). By comparison, we observed that myb75-c was more sensitive to $\mathrm{Cd}$ stress than wild type (Fig. 1C). These results were further verified by quantification of both the root length and fresh weight (Fig. 1D). Overall, these results suggest that MYB75 is involved in regulation of plant Cd tolerance.

It has previously been observed that $\mathrm{Cd}$ exposure weakens the photosystem, we next confirmed that whether MYB75 regulated Cd stress-induce photosystem damage. We maintained Arabidopsis seedlings grown in $1 / 2 \mathrm{MS}$ media without or with $75 \mu \mathrm{M} \mathrm{CdCl}_{2}$ for $21 \mathrm{~d}$. From the Fig. $2 \mathrm{~A}$ we can see that $\mathrm{Cd}$ exposure accelerated chlorophyll degradation. Quantification of chlorophyll verified that pap1-D showed more chlorophyll content compared with wild type (Fig. 2B). Meanwhile, the chlorophyll content of myb75-c mutant was significantly lower than that of wild type (Fig. 2B). Ion leakage can indicate the degree of damage in plants caused by environmental stresses, thus we analyzed the ion leakage under $\mathrm{Cd}$ exposure. As shown in Fig. 2C, ion leakage was much higher in myb75-c mutant than in wild type. Nonetheless, ion leakage of pap1-D was significantly lower than that of wild type (Fig. 2C).

We further determined the photosystem II (PSII) photochemistry by detecting Chlorophyll fluorescence including $\mathrm{F}_{\mathrm{v}} / \mathrm{F}_{\mathrm{m}}$ and ФPSII. Compared with wild type, $F_{v} / F_{m}$ of pap1-D showed no visible difference without $\mathrm{CdCl}_{2}$ (Fig. 2D). When exposed to Cd stress, pap1-D exhibited higher levels of $\mathrm{F}_{\mathrm{v}} / \mathrm{F}_{\mathrm{m}}$ than that of wild type (Fig. 2D). In contrast, levels of $F_{v} / F_{m}$ were lower in myb75-c than in wild type (Fig. 2D). Similarly, levels of ФPSII were also higher in pap 1-D and lower in myb75-c compared with wild type (Fig. 2E). These results reveal that MYB75 transcription factor alleviates Cd stress-induced photosystem damage. Together with above results, we conclude that MYB75 transcription factor participates in modulation of plant Cd tolerance.

\section{MYB75 positively regulates plant Cd tolerance}

To further examine the impact of MYB75 on plant Cd tolerance, we generated transgenic plants expressing MYB75 driven by the constitutive 35S promoter in wild type background. The 35S: MYB75 \#7 and 35S: MYB75 \#10 transgenic plants exhibited increased tolerance to $C d$ stress compared with wild type when grown on $1 / 2 \mathrm{MS}$ media with 50 or $75 \mu \mathrm{M} \mathrm{CdCl}_{2}$ (Fig. 3A). Quantitative analysis of root length 
and fresh weight further confirmed these results (Fig. 3B, C), indicating that MYB75 positively regulates plant Cd stress.

Next, the 35S: MYB75 \#7 and 35S: MYB75 \#10 transgenic plants grown on 1/2 MS media without or with $75 \mu \mathrm{M} \mathrm{CdCl}_{2}$ were examined. The chlorophyll content of 35S: MYB75 \#7 and 35S: MYB75 \#10 was significantly higher than that of wild type (Fig. 4A, B). As can be seen from Fig. 4C, ion leakage of 35S: MYB75 \#7 and 35S: MYB75 \#10 transgenic plants was significantly lower than that of wild type. When exposed to Cd stress, 35S: MYB75 \#7 and 35S: MYB75 \#10 transgenic plants exhibited higher levels of $F_{v} / F_{m}$ and $\Phi P S I l$ than that of wild type (Fig. 4D, E). Taken together, MYB75 transcription factor enhances plant $\mathrm{Cd}$ tolerance under $\mathrm{Cd}$ exposure.

\section{MYB75 transcription factor-elevated Cd tolerance is involved in antioxidant system}

Much work so far has focused on the key function of MYB75 transcription factor in regulation of anthocyanin accumulation. Given that anthocyanin is critical for scavenge ROS via their antioxidant capability (Li et al., 2017, Nakabayashi et al., 2014), we further examined the ROS levels in MYB75overexpressing lines and myb75-c mutant under normal or $\mathrm{Cd}$ stress conditions. Nitroblue tetrazolium (NBT) staining indicated that content of superoxide $\left(\mathrm{O}_{2}{ }^{-}\right)$was much lower in pap1-D, 35S: MYB75 \#7 and 35S: MYB75 \#10 plants compared with wild type under Cd stress (Fig. $5 \mathrm{~A}$ ). On the contrary, $\mathrm{O}_{2}{ }^{-{ }^{-}}$content of myb75-c was much higher than that of wild type under Cd stress (Fig. 5A). These results were further ascertained by quantification of $\mathrm{O}_{2}{ }^{--}$content (Fig. 5B). Moreover, we observed that pap1-D, 35S: MYB75 \#7 and 35S: MYB75 \#10 plants accumulated less hydrogen peroxide $\left(\mathrm{H}_{2} \mathrm{O}_{2}\right)$ content than wild type under Cd stress (Fig. 5C). In contrast, $\mathrm{H}_{2} \mathrm{O}_{2}$ content of myb75-c was much higher than that of wild type under $\mathrm{Cd}$ stress (Fig. 5C). Quantification of $\mathrm{H}_{2} \mathrm{O}_{2}$ content also confirmed these results (Fig. 5D). Collectively, these results demonstrated that MYB75 transcription factor plays positive role in protection of plants from $\mathrm{Cd}$ exposure by alleviating oxidative damage.

Extensive research has shown that plants have highly effective antioxidant mechanisms involving superoxide dismutase (SOD) and catalase (CAT) to scavenge ROS. Cd exposure led to decreases in antioxidant enzyme activities (Fig. 5E, F). It is mostly likely that $\mathrm{Cd}^{2+}$ inhibits the function of antioxidant enzyme. Intriguingly, diminution of SOD and CAT activities in myb75-c mutant were significantly aggravated under Cd exposure (Fig. 5E, F). Oppositely, SOD and CAT activities of pap1-D, 35S: MYB75 \#7 and 35S: MYB75 \#10 plants were significantly higher than that of wild type (Fig. 5E, F). In summary, MYB75 transcription factor positively regulates $\mathrm{Cd}$ tolerance through activating antioxidant system and alleviating oxidative damage.

\section{MYB75 transcription factor stimulates GSH-dependent PC synthesis pathway and Cd accumulation}


Reduced glutathione (GSH)-oxidized glutathione (GSSG) conversion by ROS homeostasis in plant cell has been intensively investigated (Noctor et al., 2012). To clarify whether ROS homeostasis affects GSH content, we examined catalase-overexpressing plants (35S: CAT2 and 35S: CAT3) under Cd exposure. When compared with wild type, 35S: CAT2 and 35S: CAT3 plants exhibited less $\mathrm{H}_{2} \mathrm{O}_{2}$ content, but more GSH and PC content under Cd stress (Fig. S2). Given that MYB75 transcription factor declined the ROS levels via anthocyanin and antioxidant enzyme, we next determined the GSH levels without or with Cd treatment. As shown in Fig. 6A, no significant difference was detected in total glutathione (GSH plus 2GSSG) between the wild type, the pap1-D, 35S: MYB75 \#7, 35S: MYB75 \#10 plants, and the myb75-c mutants without $\mathrm{Cd}$ treatment. $\mathrm{Cd}$ exposure significantly depressed GSH concentrations in these plants (Fig. 6A). Nevertheless, compared with wild type, GSH levels was higher in pap1-D, 35S: MYB75 \#7, 35S: MYB75 \#10 plants and lower in myb75-c mutants (Fig. 6A). Previous research has established that phytochelatin (PC), the important component involved in chelating $\mathrm{Cd}^{2+}$, derived directly from $\mathrm{GSH}$ via the PC synthase (PCS). The PC content was elevated significantly in pap1-D, 35S: MYB75 \#7, 35S: MYB75 \#10 plants and diminished in myb75-c mutants compared with wild type under $\mathrm{Cd}$ exposure, suggesting MYB75 transcription factor positively regulates PC levels. Taken together, these results verified that MYB75 positively regulates $\mathrm{Cd}$ tolerance through stimulating GSH-dependent PC synthesis pathway.

Given the observed change of PC, we further test whether MYB75 affects $C d$ content through measuring Cd content under $\mathrm{Cd}$ stress. As can be seen from Fig. 6C, pap1-D, 35S: MYB75 \#7, 35S: MYB75 \#10 plants showed higher $\mathrm{Cd}$ content in roots and shoots than wild type. On the contrary, Cd content was reduced in myb75-c under Cd stress compared with that in the wild type. These results suggest that MYB75 mediates $\mathrm{Cd}$ accumulation in roots and shoots under $\mathrm{Cd}$ stress.

\section{MYB75 transcription factor directly regulates the Cd tolerance-related gene expression}

Transcription factor found to be influencing Cd tolerance have been explored in several studies (Agarwal et al., 2020, Zhang et al., 2019). Therefore, we further investigated whether MYB75 regulates Cd tolerance at transcriptional level. We determined $\mathrm{Cd}$ tolerance-related gene expression such as $A C B P 2, A B C C 2$, GSH1, PDR8, ATM3, and PDF2.5. Intriguingly, the transcription levels of GSH1, PDR8, ATM3, and PDF2.5 were induced by Cd stress, but these gene expression in pap1-D, 35S: MYB75 \#7, 35S: MYB75 \#10 and myb75-c exhibited no difference compared with wild type (Fig. S3). However, under Cd stress, the transcription levels of $A C B P 2$, which binds $\mathrm{Cd}^{2+}$, were significantly higher in pap1-D, 35S: MYB75 \#7, 35S: MYB75 \#10 plants than in wild type, while its expression levels in myb75-c mutant were significantly lower than that in the wild type (Fig. 7A). We also noticed that expression levels of $A B C C 2$, an ABCC-type phytochelatin transporter, were elevated in pap1-D, 35S: MYB75 \#7, 35S: MYB75 \#10 plants, but reduced in myb75-c mutant without or with Cd stress (Fig. 7B).

On the basis of positive impact of MYB75 on $A C B P 2$ and $A B C C 2$ expression, we further surveyed the GUS activity by transient expression analysis in Nicotiana benthamiana. From the Fig. 7C-E and Fig. S4, we can see that MYB75 prompted the expression levels of ProACBP2: GUS and ProABCC2: GUS, indicating 
that MYB75 has the capacity of prompting reporter activity driven by the promoters of $A C B P 2$ and $A B C C 2$. Moreover, we performed luciferase (LUC) reporter transactivation assays in Arabidopsis protoplasts. Promoters of $A C B P 2$ and $A B C C 2$ were fused with $L U C$ gene to generate promoter-LUC reporter constructs (Fig. 7F). These reporter constructs were co-expressed with empty vector or MYB75HA in wild type protoplasts treated with MG132, and the reporter gene expression was used to evaluate MYB75 transcriptional activity. We noticed that MYB75 induced ProACBP2-LUC expression compared with empty vector (Fig. 7G). Consistently, MYB75 also induced ProABCC2-LUC expression (Fig. 7H). Based on these findings, we conclude that MYB75 positively regulates $A C B P 2$ and $A B C C 2$ expression.

\section{MYB75 transcription factor directly binds to the promoter of $A C B P 2$ and $A B C C 2$}

Taking the above observations into account, we further examined the binding of MYB75 to the promoter regions of $A C B P 2$ and $A B C C 2$ in vitro and in vivo. MYB-recognizing element (MRE) has been identified as the core binding motif of MYB75. We found two MREs within the promoters of $A C B P 2$ and $A B C C 2$ (Fig. 8A, B). To test whether MYB can directly bind to the promoter regions of these target genes, we firstly performed electrophoretic mobility shift assay (EMSA). The results revealed that MYB75 protein tagged with maltose binding protein (MBP) (MBP-MYB75) bound to the P1 probe of $A C B P 2$ and $\mathrm{C} 1$ probe $A B C C 2$, while the binding was abolished by mutation of MYB75 binding sites in the probes (Fig. 8A, B).

Interestingly, MBP-MYB75 weakly bound to the P2 probe in the promoter of $A C B P 2$, but did not bind to the $\mathrm{C} 2$ in the promoter of $A B C C 2$ (Fig. 8A, B). We next employed chromatin immunoprecipitation (ChIP) to further test the affinity of MYB75 for promoters of $A C B P 2$ and $A B C C 2$. We immunoprecipited HA-MYB75 protein from 35S: MYB75 \#7 transgenic plants treated with MG132 by using anti-GFP antibody. TA3, a retrotransposable element, was used as the internal control. The ChIP-qPCR results revealed that MYB75 significantly enriched the fragments containing P1 and P2 of ProACBP2, C1 of ProABCC2 (Fig. 8C, D). These results indicate that MYB75 directly regulates the transcription of $A C B P 2$ and $A B C C 2$ by binding to their promoters.

\section{Discussion}

As a large number of heavy metal pollutants, Cd pollution has become a global crisis with industrial development, causes serious environmental damage and human disease. High concentration of $\mathrm{Cd}$ in the soil also inhibits plant growth and crop yield. In recent years, there has been an increasing interest in studying the mechanism by which plants enrich and detoxify $\mathrm{Cd}$. In this study, we established that MYB75 transcription factor positively regulates plant Cd stress via multiple pathways. Our research revealed that MYB75 stimulates GSH and PC content and activates of Cd tolerance-related gene at transcriptional level, such as $A C B P 2$ and $A B C C 2$, thus inspiring a new transcription factor function in elevating plant $\mathrm{Cd}$ tolerance. 


\section{MYB75 positively regulates plant Cd tolerance via anthocyanin}

It is well established that anthocyanin accumulation is induced by extreme environmental factors, such as high light, drought, cold, thus protecting plants against ROS damage (Allan et al., 2018). Several studies suggested that $\mathrm{Cd}$ stress stimulated anthocyanin accumulation. Anthocyanin biosynthetic genes transcription were induced under Cd exposure (Dobrikova et al., 2021, Mwamba et al., 2020). As we all know, anthocyanin biosynthetic genes were regulated by MBW protein complex. It seems that Cd stress affected anthocyanin regulatory genes expression. Our results illuminated that $\mathrm{Cd}$ exposure primarily induce MYB75 expression, thus prompting MBW protein complex and stimulating anthocyanin accumulation (Fig. 1A, B). These findings reveal that Cd exposure aggravated anthocyanin accumulation via transcriptional regulation of MBW protein complex.

Several researches demonstrated that there was a negative correlation between the chlorophyll and anthocyanin content under Cd stress (Szopinski et al., 2020, Vazquez et al., 2020). We found that pap1-D, 35S: MYB75 \#7 and 35S: MYB75 \#10, which accumulated abundant anthocyanin, exhibited higher chlorophyll level and fluorescence than wild type (Fig. 2 and 3). These results imply that anthocyanin can protect photosystem from ROS damage under Cd exposure. It has previously been observed that exogenous anthocyanin increased intracellular GSH levels (Norris et al., 2016). We verified that endogenous anthocyanin elevated GSH and PC levels through modulating ROS homeostasis (Fig. 6A, B). Our results are also in accord with previous studies indicating that anthocyanin is beneficial to Cd uptake and hyperaccumulate Cd under Cd exposure (Mwamba et al., 2020, Szopinski et al., 2020) (Fig. 6C). Previous studies showed that anthocyanins and organic acids can facilitate localization of Cd into vacuole (Glinska et al., 2013, Sebastian et al., 2018, Verbruggen et al., 2009). We speculated that endogenous anthocyanins can benefit $\mathrm{Cd}^{2+}$ sequestration to reduce $\mathrm{Cd}$ toxicity. These observations may support the hypothesis that plants adaptively prompt anthocyanin accumulation during suffering $\mathrm{Cd}$ stress thus scavenging ROS and expediting $\mathrm{Cd}^{2+}$ sequestration to alleviate $\mathrm{Cd}$ toxicity.

\section{MYB75 enhances plant Cd tolerance at transcriptional level}

Under Cd exposure, plants evolve an adaptive mechanism by which transcription factors regulate expression of numerous $\mathrm{Cd}$ tolerance-related genes at transcriptional level. ZAT6 regulates $\mathrm{Cd}$ tolerance by upregulating PC synthesis-related genes (Chen et al., 2016). MYB49, the R2R3-MYB transcription factor, results $\mathrm{Cd}$ accumulation through inducing HIPP22 and HIPP44 transcription (Zhang et al., 2019). Cd-induced WRKY13 activates $D C D$ expression to increase the production of $\mathrm{H}_{2} \mathrm{~S}$, brings about higher $\mathrm{Cd}$ tolerance (Zhang et al., 2020). In our study, LUC and GUS staining assays suggested that MYB75 reinforced the activities of the promoters of $A C B P 2$ and $A B C C 2$ (Fig. $7 \mathrm{C}-\mathrm{H}$ ). EMSA assays demonstrated that MYB75 directly bound to the MYB-recognizing element in the promoter of $A C B P 2$ and $A B C C 2$ (Fig. 8A, B). ChIP-qPCR assays proved that $\mathrm{Cd}$ stress strengthened binding of MYB75 to $A C B P 2$ and $A B C C 2$ (Fig. $8 \mathrm{C}, \mathrm{D}$ ). These results imply that MYB75 directly regulates the $A C B P 2$ and $A B C C 2$ expression. 
Previous studies confirmed that ACBP2 alleviates Cd toxicity through binding $\mathrm{Cd}^{2+}$ (Gao et al., 2010). Additionally, $\mathrm{ABA}$ and drought induces $A C B P 2$ transcription thus enhancing drought tolerance (Du et al., 2013). Intriguingly, anthocyanin accumulation is raised by ABA and drought. It seems that MYB75 was activated upon drought stress and then elevated $A C B P 2$ expression to improve drought tolerance. These findings suggested that MYB75 perhaps participated in diversified stress response. Prior reports have shown that ABCC2 exhibited PC transport activity in plant vacuole (Song et al., 2010). Subsequent research observed that anthocyanin is transported to vacuole via ABCC2 (Behrens et al., 2019). It can thus be suggested that vacuole played an important role in detoxifying $\mathrm{Cd}$ via $\mathrm{ABCC}$-modulated $\mathrm{Cd}^{2+}$ sequestration. According to these data, we infer that regulation of MYB75 on $\mathrm{Cd}^{2+}$ sequestration and compartmentation at transcriptional and metabolic level enhances plant Cd tolerance.

\section{MYB75 balance growth and the stress response}

Recent studies suggested that reciprocal regulation between stress-response and growth-control pathways occurs at multiple levels. Plants need to strictly limit the allocation of carbon sources to balance development and extreme environmental response. Extensive research has shown that MYB75 functions in regulation of secondary cell wall formation during plant development (Bhargava et al., 2010). MYB75 transcription facilitates carbon flow into the anthocyanin pathway rather than lignin pathway under various environmental stresses. Our previous studies demonstrated plant fine tune anthocyanin accumulation via post-translational regulation of MYB75 through HAT1 transcription factor and SUMO E3 ligase SIZ1 during growth (Zheng et al., 2020b, Zheng et al., 2019). In general, therefore, it seems that MYB75 primarily regulates secondary cell wall formation under normal condition. Once plant suffers stress including $\mathrm{Cd}$ and drought, the activities of MYB75 is enhanced via multiple levels of regulation, thus promoting anthocyanin accumulation to adapt to the extreme environment.

In conclusion, a working model was proposed based on our study (Fig. 9). When the plants are confronted with Cd stress, expression of MYB75 transcription factor could be quickly induced. Cd-induced MYB75 stimulates GSH-dependent PC synthesis via anthocyanin-mediated ROS homeostasis. On the other side, MYB75 activates $A C B P 2$ and $A B C C 2$ expression by directly binding to the promoters of $A C B P 2$ and $A B C C 2$, followed by $C d$ sequestration and compartmentation, thus enhancing plant $\mathrm{Cd}$ tolerance.

\section{Declarations}

\section{Author contributions}

D.W.Z conceived and supervised the study. T.Z and F.Y designed experiments and analyzed data. T.Z performed experiments. T.Z and D.W.Z wrote the manuscript.

\section{Acknowledgments}

The pap1-D and myb75-c were kindly provided by Prof. Daoxin Xie and Prof. Jinlong Qiu respectively. The 35S: CAT2 and 35S: CAT3 were kindly provided by Prof Mingyi Bai. This work was financially supported 
by the grants from the National Natural Science Foundation of China (http://www.nsfc.gov.cn/) [32100240 to TZ]; the project of Starting Research Fund from Sichuan Normal University [024341977001].

\section{Declaration of Competing Interest}

The authors have declared that no competing interests exist.

\section{References}

1. Agarwal P, Mitra M, Banerjee S, Roy S (2020) MYB4 transcription factor, a member of R2R3subfamily of MYB domain protein, regulates cadmium tolerance via enhanced protection against oxidative damage and increases expression of PCS1 and MT1C in Arabidopsis. Plant Sci 297

2. Allan AC, Espley RV (2018) MYBs Drive Novel Consumer Traits in Fruits and Vegetables. Trends Plant Sci 23: 693-705

3. Baker NR (2008) Chlorophyll fluorescence: a probe of photosynthesis in vivo. Annu Rev Plant Biol 59: 89-113

4. Behrens CE, Smith KE, lancu CV, Choe JY, Dean JV (2019) Transport of Anthocyanins and other Flavonoids by the Arabidopsis ATP-Binding Cassette Transporter AtABCC2. Sci Rep 9: 437

5. Bhargava A, Mansfield SD, Hall HC, Douglas CJ, Ellis BE (2010) MYB75 functions in regulation of secondary cell wall formation in the Arabidopsis inflorescence stem. Plant Physiol 154: 1428-38

6. Borevitz JO, Xia Y, Blount J, Dixon RA, Lamb C (2000) Activation tagging identifies a conserved MYB regulator of phenylpropanoid biosynthesis. Plant Cell 12: 2383-2394

7. Chang JD, Huang S, Konishi N, Wang P, Chen J, Huang XY, Ma JF, Zhao FJ (2020) Overexpression of the manganese/cadmium transporter OsNRAMP5 reduces cadmium accumulation in rice grain. $J$ Exp Bot 71: 5705-5715

8. Chen J, Yang LB, Yan XX, Liu YL, Wang R, Fan TT, Ren YB, Tang XF, Xiao FM, Liu YS, Cao SQ (2016) Zinc-Finger Transcription Factor ZAT6 Positively Regulates Cadmium Tolerance through the Glutathione-Dependent Pathway in Arabidopsis. Plant Physiology 171: 707-719

9. Clemens S (2019) Safer food through plant science: reducing toxic element accumulation in crops. $J$ Exp Bot 70: 5537-5557

10. Clemens S, Ma JF (2016) Toxic Heavy Metal and Metalloid Accumulation in Crop Plants and Foods. Annu Rev Plant Biol 67: 489-512

11. Cuypers A, Plusquin $M$, Remans $T$, Jozefczak $M$, Keunen E, Gielen $H$, Opdenakker $K$, Nair AR, Munters E, Artois TJ, Nawrot T, Vangronsveld J, Smeets K (2010) Cadmium stress: an oxidative challenge. Biometals 23: 927-40

12. Dai LP, Dong XJ, Ma HH (2012) Molecular mechanism for cadmium-induced anthocyanin accumulation in Azolla imbricata. Chemosphere 87: 319-25 
13. Ding Y, Jia Y, Shi Y, Zhang X, Song C, Gong Z, Yang S (2018) OST1-mediated BTF3L phosphorylation positively regulates $\mathrm{CBFs}$ during plant cold responses. $E M B O \mathrm{~J} 37$

14. Dobrikova AG, Apostolova EL, Hanc A, Yotsova E, Borisova P, Sperdouli I, Adamakis IS, Moustakas M (2021) Cadmium toxicity in Salvia sclarea L.: An integrative response of element uptake, oxidative stress markers, leaf structure and photosynthesis. Ecotoxicol Environ Saf 209: 111851

15. Du ZY, Chen MX, Chen QF, Xiao S, Chye ML (2013) Overexpression of Arabidopsis acyl-CoA-binding protein ACBP2 enhances drought tolerance. Plant Cell Environ 36: 300-14

16. Gao W, Li HY, Xiao S, Chye ML (2010) Acyl-CoA-binding protein 2 binds lysophospholipase 2 and lysoPC to promote tolerance to cadmium-induced oxidative stress in transgenic Arabidopsis. Plant $J$ 62: $989-1003$

17. Gao W, Xiao S, Li HY, Tsao SW, Chye ML (2009) Arabidopsis thaliana acyl-CoA-binding protein ACBP2 interacts with heavy-metal-binding farnesylated protein AtFP6. New Phytol 181: 89-102

18. Glinska S, Gapinska M (2013) The effect of pre-incubation of Allium cepa L. roots in the ATH-rich extract on Pb uptake and localization. Protoplasma 250: 601-11

19. González A, Laporte D, Moenne A (2021) Cadmium Accumulation Involves Synthesis of Glutathione and Phytochelatins, and Activation of CDPK, CaMK, CBLPK, and MAPK Signaling Pathways in Ulva compressa. Frontiers in Plant Science 12

20. Haider FU, Liqun C, Coulter JA, Cheema SA, Wu J, Zhang R, Wenjun M, Farooq M (2021) Cadmium toxicity in plants: Impacts and remediation strategies. Ecotoxicol Environ Saf 211: 111887

21. Han YY, Fan TT, Zhu XY, Wu X, Ouyang J, Jiang L, Cao SQ (2019) WRKY12 represses GSH1 expression to negatively regulate cadmium tolerance in Arabidopsis. Plant Molecular Biology 99: 149-159

22. He YQ, Zhang XY, Li LY, Sun ZT, Li JM, Chen XY, Hong GJ (2021) SPX4 interacts with both PHR1 and PAP1 to regulate critical steps in phosphorus-status-dependent anthocyanin biosynthesis. New Phytol 230: 205-217

23. Herbette S, Taconnat L, Hugouvieux V, Piette L, Magniette ML, Cuine S, Auroy P, Richaud P, Forestier C, Bourguignon J, Renou JP, Vavasseur A, Leonhardt N (2006) Genome-wide transcriptome profiling of the early cadmium response of Arabidopsis roots and shoots. Biochimie 88: 1751-65

24. Huybrechts M, Cuypers A, Deckers J, Iven V, Vandionant S, Jozefczak M, Hendrix S (2019) Cadmium and Plant Development: An Agony from Seed to Seed. Int J Mol Sci 20

25. Lei X, Liu WH, Zhao JM, You MH, Xiong CH, Xiong Y, Xiong YL, Yu QQ, Bai SQ, Ma X (2020) Comparative Physiological and Proteomic Analysis Reveals Different Involvement of Proteins during Artificial Aging of Siberian Wildrye Seeds. Plants-Base/ 9

26. Li P, Li YJ, Zhang FJ, Zhang GZ, Jiang XY, Yu HM, Hou BK (2017) The Arabidopsis UDPglycosyltransferases UGT79B2 and UGT79B3, contribute to cold, salt and drought stress tolerance via modulating anthocyanin accumulation. Plant J 89: 85-103

27. Li QF, He JX (2016) BZR1 Interacts with HY5 to Mediate Brassinosteroid- and Light-Regulated Cotyledon Opening in Arabidopsis in Darkness. Mol Plant 9: 113-125 
28. Luo JS, Yang Y, Gu T, Wu Z, Zhang Z (2019) The Arabidopsis defensin gene AtPDF2.5 mediates cadmium tolerance and accumulation. Plant Cell Environ 42: 2681-2695

29. Mwamba TM, Islam F, Ali B, Lwalaba JLW, Gill RA, Zhang F, Farooq MA, Ali S, Ulhassan Z, Huang Q, Zhou W, Wang J (2020) Comparative metabolomic responses of low- and high-cadmium accumulating genotypes reveal the cadmium adaptive mechanism in Brassica napus. Chemosphere 250: 126308

30. Nakabayashi R, Yonekura-Sakakibara K, Urano K, Suzuki M, Yamada Y, Nishizawa T, Matsuda F, Kojima M, Sakakibara H, Shinozaki K, Michael AJ, Tohge T, Yamazaki M, Saito K (2014) Enhancement of oxidative and drought tolerance in Arabidopsis by overaccumulation of antioxidant flavonoids. Plant J 77: 367-79

31. Noctor G, Mhamdi A, Chaouch S, Han Y, Neukermans J, Marquez-Garcia B, Queval G, Foyer CH (2012) Glutathione in plants: an integrated overview. Plant Cell Environ 35: 454-84

32. Norris KM, Okie W, Yakaitis CL, Pazdro R (2016) The anthocyanin cyanidin-3-O-beta-glucoside modulates murine glutathione homeostasis in a manner dependent on genetic background. Redox Biol 9: 254-263

33. Sebastian A, Prasad MNV (2018) Exogenous citrate and malate alleviate cadmium stress in Oryza sativa L.: Probing role of cadmium localization and iron nutrition. Ecotoxicol Environ Saf 166: 215222

34. Sheng Y, Yan X, Huang Y, Han Y, Zhang C, Ren Y, Fan T, Xiao F, Liu Y, Cao S (2019) The WRKY transcription factor, WRKY13, activates PDR8 expression to positively regulate cadmium tolerance in Arabidopsis. Plant Cell Environ 42: 891-903

35. Song WY, Park J, Mendoza-Cozatl DG, Suter-Grotemeyer M, Shim D, Hortensteiner S, Geisler M, Weder B, Rea PA, Rentsch D, Schroeder JI, Lee Y, Martinoia E (2010) Arsenic tolerance in Arabidopsis is mediated by two ABCC-type phytochelatin transporters. P Natl Acad Sci USA 107: 21187-21192

36. Szopinski M, Sitko K, Rusinowski S, Zieleznik-Rusinowska P, Corso M, Rostanski A, Rojek-Jelonek M, Verbruggen N, Malkowski E (2020) Different strategies of Cd tolerance and accumulation in Arabidopsis halleri and Arabidopsis arenosa. Plant Cell Environ 43: 3002-3019

37. Vazquez A, Recalde L, Cabrera A, Groppa MD, Benavides MP (2020) Does nitrogen source influence cadmium distribution in Arabidopsis plants? Ecotox Environ Safe 191

38. Verbruggen $\mathrm{N}$, Hermans $\mathrm{C}$, Schat $\mathrm{H}$ (2009) Molecular mechanisms of metal hyperaccumulation in plants. New Phytol 181: 759-776

39. Waszczak C, Carmody M, Kangasjarvi J (2018) Reactive Oxygen Species in Plant Signaling. Annu Rev Plant Biol 69: 209-236

40. Wong CKE, Cobbett CS (2009a) HMA P-type ATPases are the major mechanism for root-to-shoot Cd translocation in Arabidopsis thaliana. New Phytol 181: 71-78

41. Wong CKE, Jarvis RS, Sherson SM, Cobbett CS (2009b) Functional analysis of the heavy metal binding domains of the Zn/Cd-transporting ATPase, HMA2, in Arabidopsis thaliana. New Phyto/ 181: 79-88 
42. Wu X, Han Y, Zhu X, Shah A, Wang W, Sheng Y, Fan T, Cao S (2019) Negative regulation of cadmium tolerance in Arabidopsis by MMDH2. Plant Mol Biol 101: 507-516

43. Wu X, Su NN, Yue XM, Fang B, Zou JW, Chen YH, Shen ZG, Cui J (2021) IRT1 and ZIP2 were involved in exogenous hydrogen-rich water-reduced cadmium accumulation in Brassica chinensis and Arabidopsis thaliana. $J$ Hazard Mater 407

44. Xu W, Dubos C, Lepiniec L (2015) Transcriptional control of flavonoid biosynthesis by MYB-bHLHWDR complexes. Trends Plant Sci 20: 176-85

45. Yoo SD, Cho YH, Sheen J (2007) Arabidopsis mesophyll protoplasts: a versatile cell system for transient gene expression analysis. Nat Protoc 2: 1565-72

46. Yu X, Li L, Li L, Guo M, Chory J, Yin Y (2008) Modulation of brassinosteroid-regulated gene expression by Jumonji domain-containing proteins ELF6 and REF6 in Arabidopsis. Proc Natl Acad Sci U S A 105: 7618-23

47. Zhang D, Tan W, Yang F, Han Q, Deng X, Guo H, Liu B, Yin Y, Lin H (2021) A BIN2-GLK1 Signaling Module Integrates Brassinosteroid and Light Signaling to Repress Chloroplast Development in the Dark. Dev Cell 56: 310-324 e7

48. Zhang DW, Xu F, Zhang ZW, Chen YE, Du JB, Jia SD, Yuan S, Lin HH (2010) Effects of light on cyanide-resistant respiration and alternative oxidase function in Arabidopsis seedlings. Plant Cell Environ 33: 2121-31

49. Zhang P, Wang R, Ju Q, Li W, Tran LP, Xu J (2019) The R2R3-MYB Transcription Factor MYB49 Regulates Cadmium Accumulation. Plant Physio/ 180: 529-542

50. Zhang Q, Cai W, Ji TT, Ye L, Lu YT, Yuan TT (2020) WRKY13 Enhances Cadmium Tolerance by Promoting D-CYSTEINE DESULFHYDRASE and Hydrogen Sulfide Production. Plant Physiol 183: 345357

51. Zheng T, Li Y, Lei W, Qiao K, Liu B, Zhang D, Lin H (2020a) SUMO E3 Ligase SIZ1 stabilizes MYB75 to regulate anthocyanin accumulation under high light conditions in Arabidopsis. Plant Sci 292: 110355

52. Zheng T, Li YL, Lei W, Qiao K, Liu BH, Zhang DW, Lin HH (2020b) SUMO E3 Ligase SIZ1 stabilizes MYB75 to regulate anthocyanin accumulation under high light conditions in Arabidopsis. Plant Sci 292

53. Zheng T, Tan W, Yang H, Zhang L, Li T, Liu B, Zhang D, Lin H (2019) Regulation of anthocyanin accumulation via MYB75/HAT1/TPL-mediated transcriptional repression. PLoS Genet 15: e1007993

54. Zou LJ, Deng XG, Han XY, Tan WR, Zhu LJ, Xi DH, Zhang DW, Lin HH (2016) Role of Transcription Factor HAT1 in Modulating Arabidopsis thaliana Response to Cucumber mosaic virus. Plant Cell Physiol 57: 1879-89

55. Zou MM, Zhou SL, Zhou YJ, Jia ZY, Guo TW, Wang JX (2021) Cadmium pollution of soil-rice ecosystems in rice cultivation dominated regions in China: A review. Environ Pollut 280

\section{Figures}


A

B

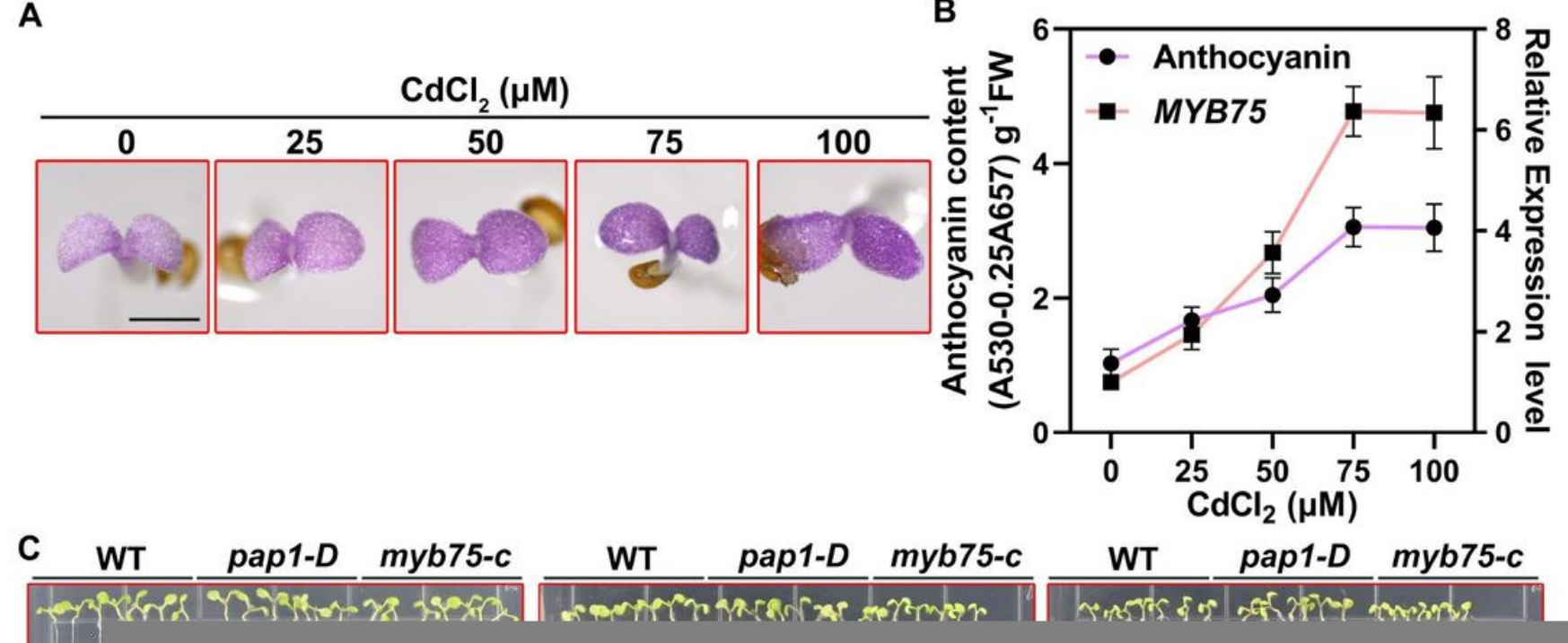

\section{Figure 1}

MYB75 transcription factor is involved in plant Cd tolerance. (A) 3-day-old WT Arabidopsis seedlings grown on plates under different Cd stress. Bars $=0.25 \mathrm{~cm}$. (B) Anthocyanin levels and MYB75 transcripts in extracts from seedlings in (A). The experiments were performed in biological triplicate (representing anthocyanin content measured from $0.2 \mathrm{~g}$ WT plants and treatment were pooled for one replicate). FW, fresh weight. Error bars denote \pm SD $(n=3)$. (C) Phenotypes of WT, pap1-Dand myb75-c mutants under Cd stress. 3-day-old seedlings grown on 1/2 MS medium were transferred to 1/2 MS medium without or with 50 or $75 \mu \mathrm{M} \mathrm{CdCl}_{2}$. Photographs were taken $7 \mathrm{~d}$ after transfer. Bar $=1 \mathrm{~cm}$. (D) Root length of seedlings described in $(C)$. Data are means $\pm S D ; n=26$ biologically independent roots. Different letters represented 
statistically significant differences (two-way ANOVA, $p<0.05$ ). (E) Fresh weight of seedlings described in C. Five plants per genotype from one plate were measured for each repeat. Data are means $\pm S D(n=3)$. Different letters represented statistically significant differences (two-way ANOVA, $p<0.05$ ).

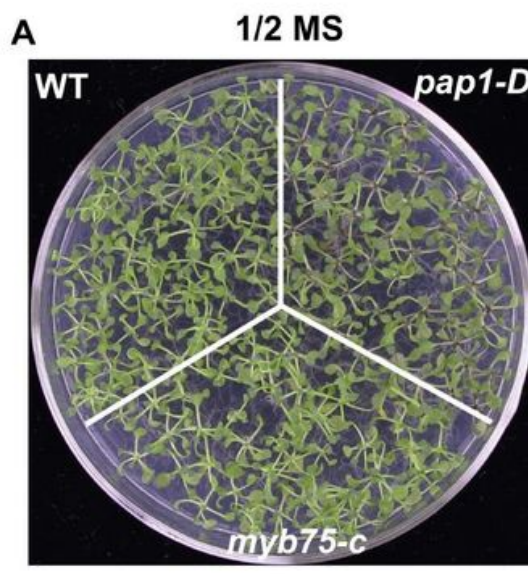

C

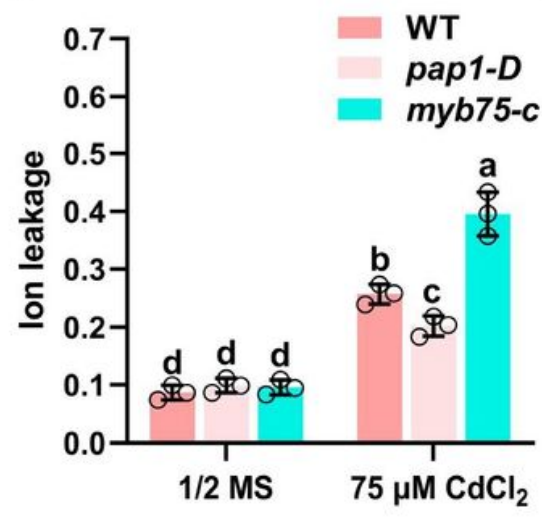

$75 \mu \mathrm{M} \mathrm{CdCl}_{2}$

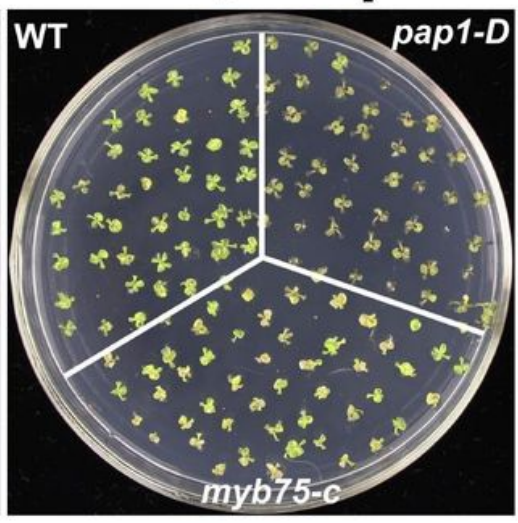

D

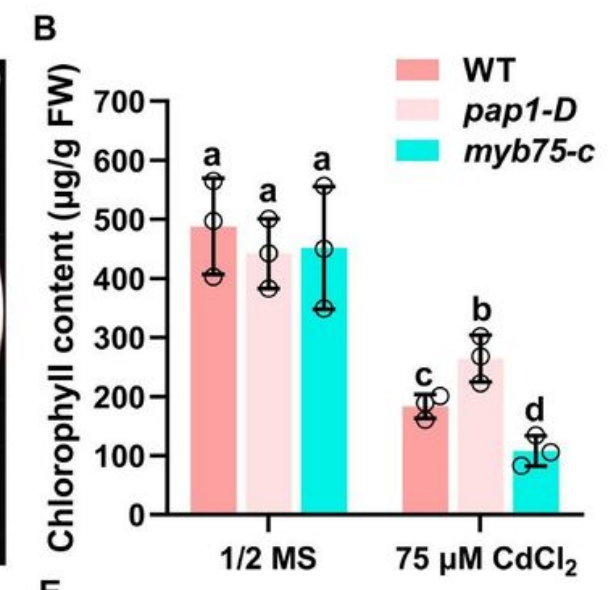

E

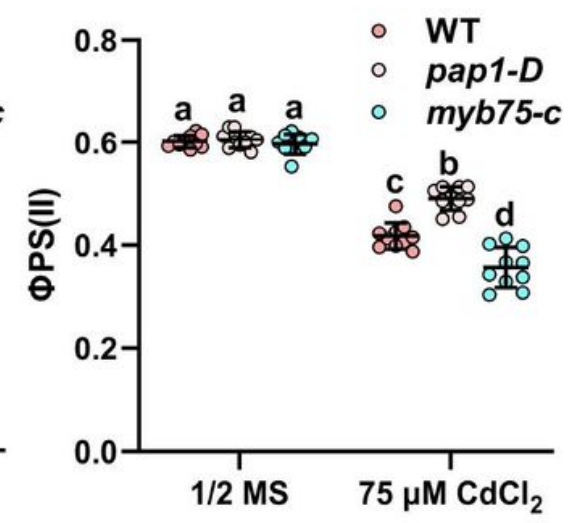

Figure 2

MYB75 enhances plant Cd tolerance. (A) 21-day-old Arabidopsis seedlings of WT, pap1-D, myb75-c grown on plates under different Cd stress. (B) Chlorophyll levels in extracts from seedlings in (A). The experiments were performed in biological triplicate (representing chlorophyll content measured from 15 plants of each genotype and treatment were pooled for one replicate). FW, fresh weight. Error bars denote $\pm S D(n=3)$. Different letters represented statistically significant differences (two-way ANOVA, $p<0.05)$. (C) Ion leakage of the plants in (A). Error bars denote $\pm S D(n=3)$. (D) $F_{v} / F_{m}$ of seedlings described in $(A)$. Data are means $\pm S D ; n=10$ biologically independent seedlings. Different letters represented statistically significant differences (two-way ANOVA, $p<0.05$ ). (E) ФPS(II) of seedlings described in (A). Data are means $\pm S D ; n=10$ biologically independent seedlings. Different letters represented statistically significant differences (two-way ANOVA, $p<0.05$ ). 


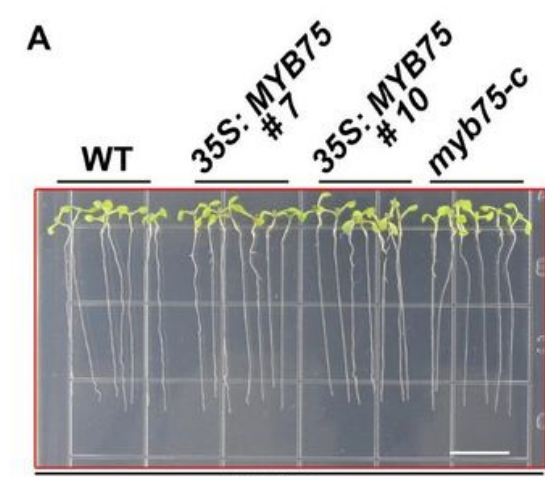

$1 / 2 \mathrm{MS}$

B

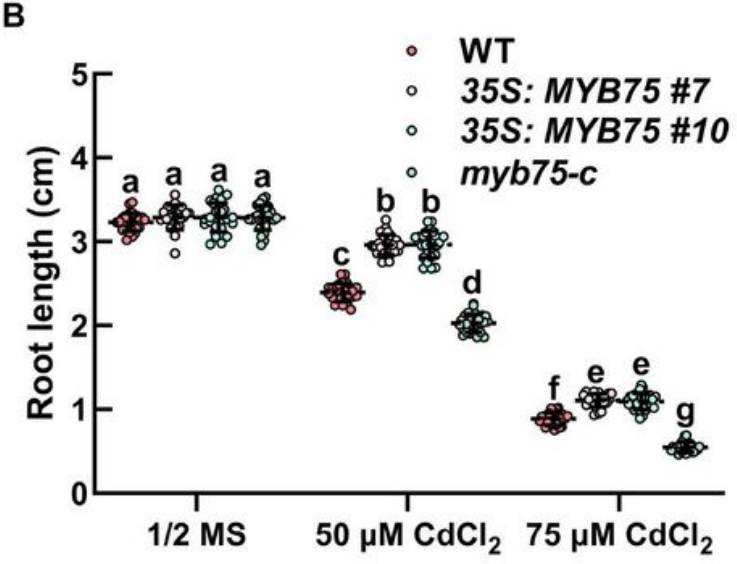

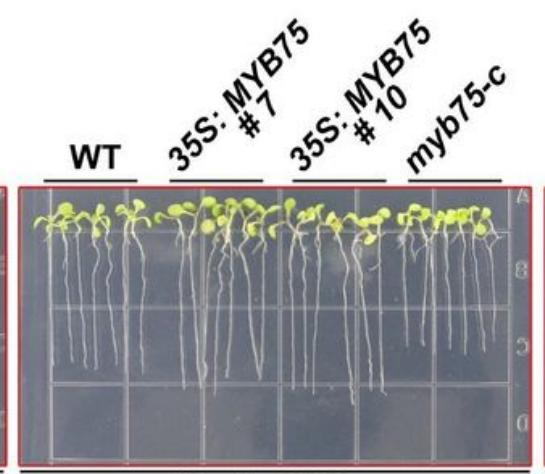

$50 \mu \mathrm{M} \mathrm{CdCl}_{2}$

C
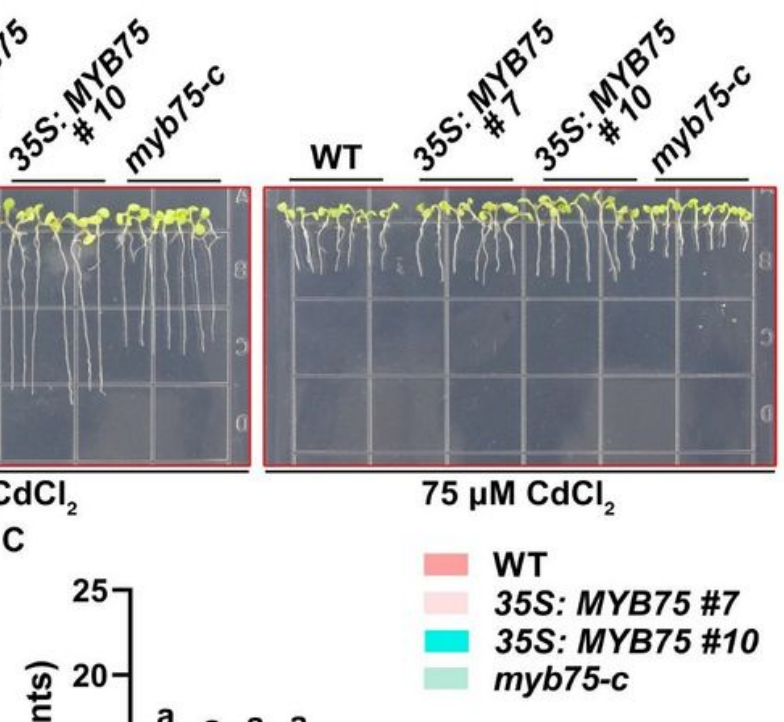

$75 \mu \mathrm{M} \mathrm{CdCl}_{2}$

WT myb75-c

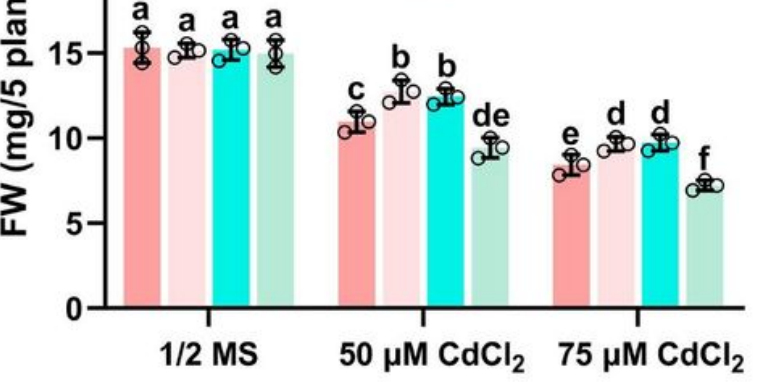

\section{Figure 3}

Cd tolerance in the MYB75 overexpression lines. (A) Phenotypes of WT, 35S: MYB75 \#7, 35S: MYB75 \#10 and myb75-c mutants under Cd stress. Three-day-old seedlings grown on 1/2 MS medium were transferred to $1 / 2 \mathrm{MS}$ medium without or with 50 or $75 \mu \mathrm{M} \mathrm{CdCl} 2$. Photographs were taken $7 \mathrm{~d}$ after transfer. Bar $=1 \mathrm{~cm}$. (B) Root length of seedlings described in (A). Data are means $\pm S D ; n=26$ biologically independent roots. Different letters represented statistically significant differences (two-way ANOVA, $p<0.05$ ). (C) Fresh weight of seedlings described in A. Five plants per genotype from one plate were measured for each repeat. Data are means $\pm S D(n=3)$. Different letters represented statistically significant differences (two-way ANOVA, $p<0.05$ ). 

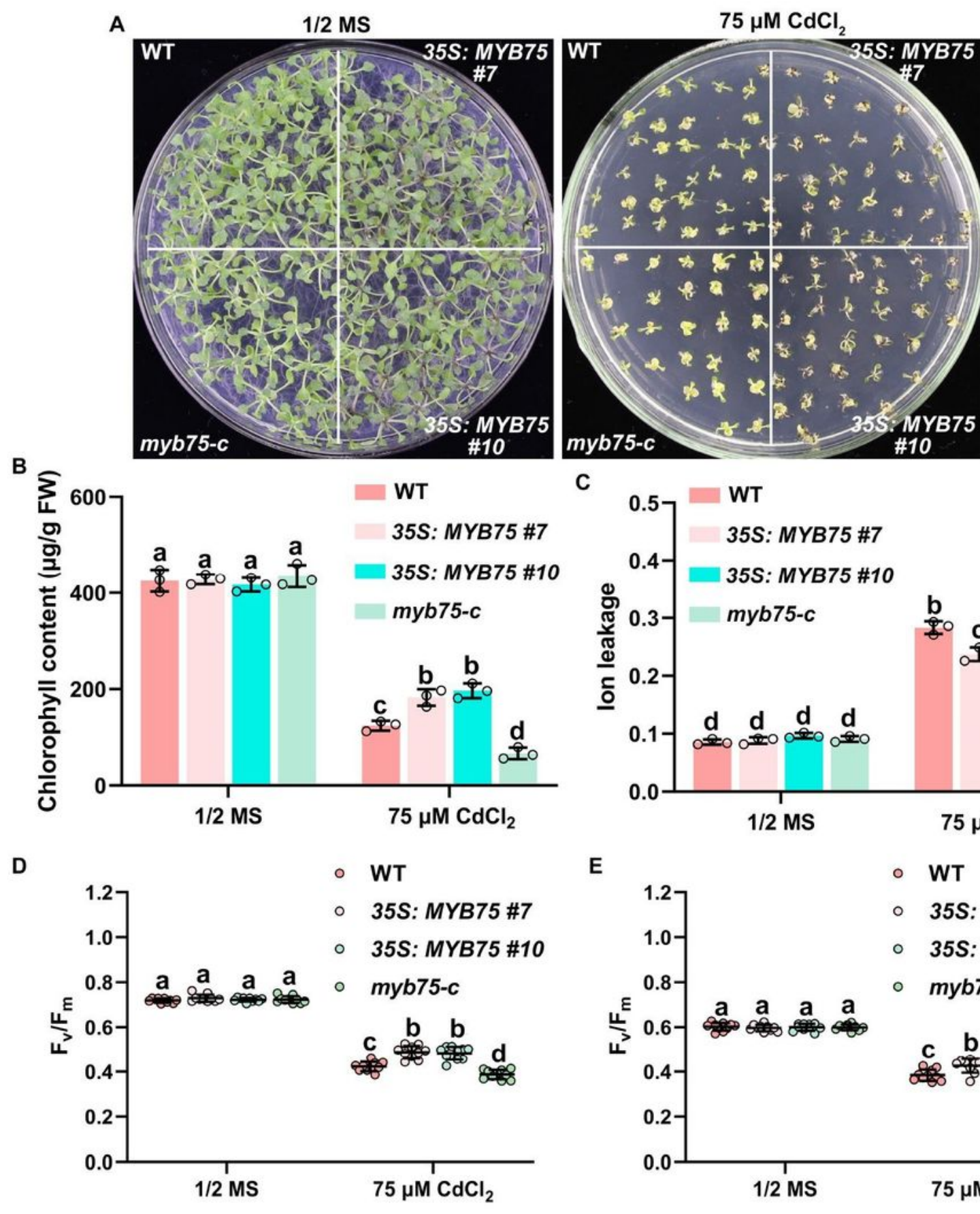

C

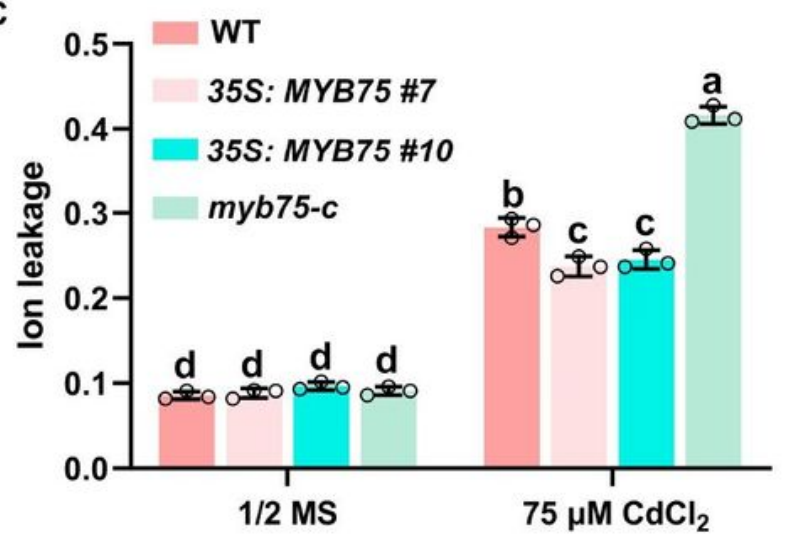

E

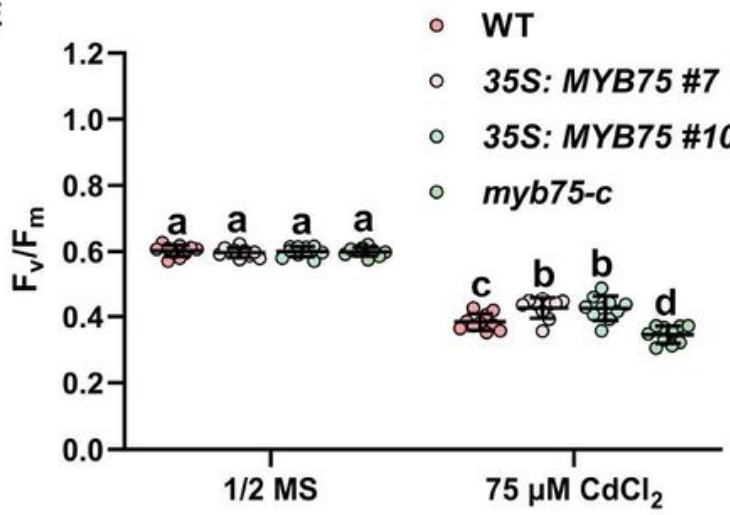

Figure 4

MYB75 overexpression enhances plant Cd tolerance (A) 21-day-old Arabidopsis seedlings of WT, 35S: MYB75 \#7, 35S: MYB75 \#10 and myb75-c grown on plates under different Cd stress. (B) Chlorophyll levels in extracts from seedlings in (A). The experiments were performed in biological triplicate (representing chlorophyll content measured from 15 plants of each genotype and treatment were pooled for one replicate). FW, fresh weight. Error bars denote $\pm S D(n=3)$. Different letters represented statistically 
significant differences (two-way ANOVA, $p<0.05$ ). (C) lon leakage of the plants in (A). Error bars denote \pm $S D(n=3)$. (D) $F_{v} / F_{m}$ of seedlings described in (A). Data are means $\pm S D ; n=10$ biologically independent seedlings. Different letters represented statistically significant differences (two-way ANOVA, $p<0.05)$. (E) ФPS(II) of seedlings described in (A). Data are means \pm SD; $n=10$ biologically independent seedlings. Different letters represented statistically significant differences (two-way ANOVA, $p<0.05$ ).
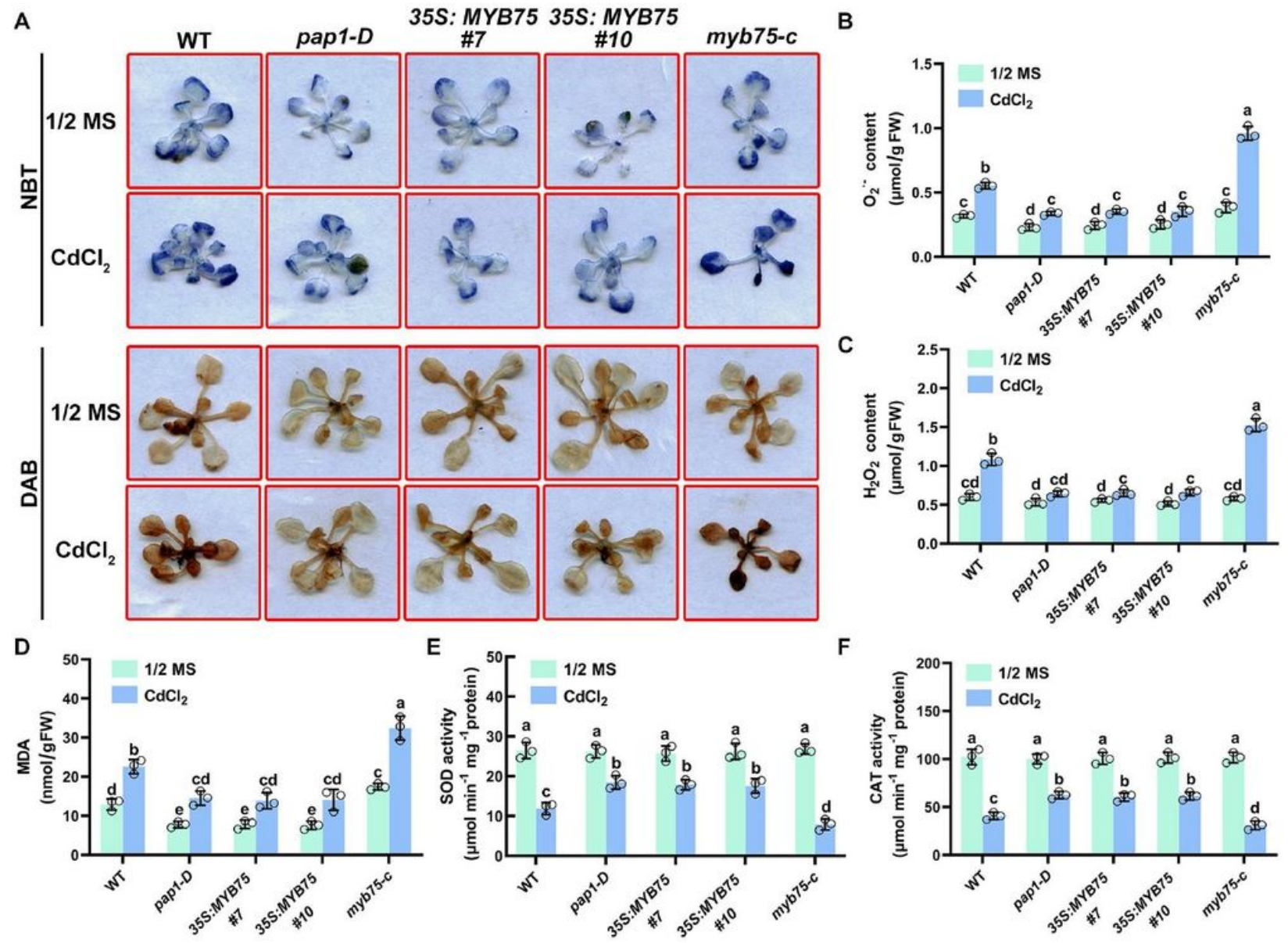

\section{Figure 5}

MYB75 modulates ROS homeostasis under Cd stress. (A) Representative images of NBT-stained and DAB-stained leaves from WT, pap1-D, 35S: MYB75 \#7, 35S: MYB75 \#10 and myb75-c seedlings grown on medium for 2 weeks and then treated with (+) or without (-) $75 \mu \mathrm{M} \mathrm{CdCl}_{2}$ for $24 \mathrm{~h}$. (B) $\mathrm{O}_{2}$ - content in extracts from seedlings in (A). The experiments were performed in biological triplicate (representing $\mathrm{O}_{2} \cdot-$ content measured from 10 plants of each genotype and treatment were pooled for one replicate). FW, fresh weight. Error bars denote $\pm S D(n=3)$. Different letters represented statistically significant differences (two-way ANOVA, $p<0.05$ ). (C) $\mathrm{H}_{2} \mathrm{O}_{2}$ content in extracts from seedlings in (A). The experiments were performed in biological triplicate (representing $\mathrm{H}_{2} \mathrm{O}_{2}$ content measured from 10 plants of each 
genotype and treatment were pooled for one replicate). FW, fresh weight. Error bars denote $\pm S D(n=3)$. Different letters represented statistically significant differences (two-way ANOVA, $p<0.05$ ). (D) MDA contents in extracts from seedlings in (A). The experiments were performed in biological triplicate (representing MDA content measured from 10 plants of each genotype and treatment were pooled for one replicate). FW, fresh weight. Error bars denote $\pm S D(n=3)$. Different letters represented statistically significant differences (two-way ANOVA, $p<0.05$ ). (E) SOD activity in extracts from seedlings in (A). Error bars denote $\pm S D(n=3)$. Different letters represented statistically significant differences (two-way ANOVA, $p<0.05)$. (F) CAT activity in extracts from seedlings in $(A)$. Error bars denote $\pm S D(n=3)$. Different letters represented statistically significant differences (two-way ANOVA, $p<0.05$ ).
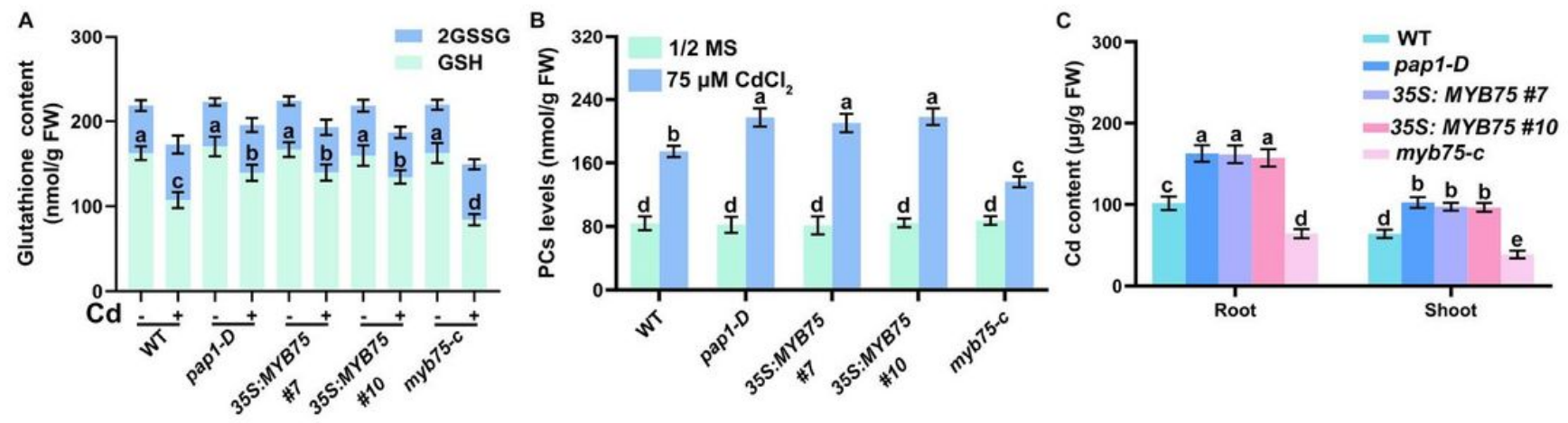

\section{Figure 6}

MYB75 mediates GSH, PCs levels and Cd distribution. (A) Glutathione contents in WT, pap1-D, 35S: MYB75 \#7, 35S: MYB75 \#10 and myb75-c seedlings grown on medium for 2 weeks and then treated with (+) or without (-) $75 \mu \mathrm{M} \mathrm{CdCl}_{2}$ for $24 \mathrm{~h}$. The experiments were performed in biological triplicate (representing glutathione content measured from 10 plants of each genotype and treatment were pooled for one replicate). $F W$, fresh weight. Error bars denote $\pm S D(n=3)$. Different letters represented statistically significant differences (two-way ANOVA, p<0.05). (B) PC contents in WT, pap1-D, 35S: MYB75 \#7, 35S: MYB75 \#10 and myb75-c seedlings grown on medium for 2 weeks and then treated with or without 75 $\mu \mathrm{M} \mathrm{CdCl}_{2}$ for $24 \mathrm{~h}$. The experiments were performed in biological triplicate (representing PC content measured from 10 plants of each genotype and treatment were pooled for one replicate). FW, fresh weight. Error bars denote $\pm S D(n=3)$. Different letters represented statistically significant differences (two-way ANOVA, p<0.05). (C) Cd contents in WT, pap1-D, 35S: MYB75 \#7, 35S: MYB75 \#10 and myb75-c seedlings grown on medium for 2 weeks. The experiments were performed in biological triplicate (representing $\mathrm{Cd}$ content measured from 10 plants of each genotype and treatment were pooled for one replicate). FW, fresh weight. Error bars denote $\pm S D(n=3)$. Different letters represented statistically significant differences (two-way ANOVA, $p<0.05$ ). 
A

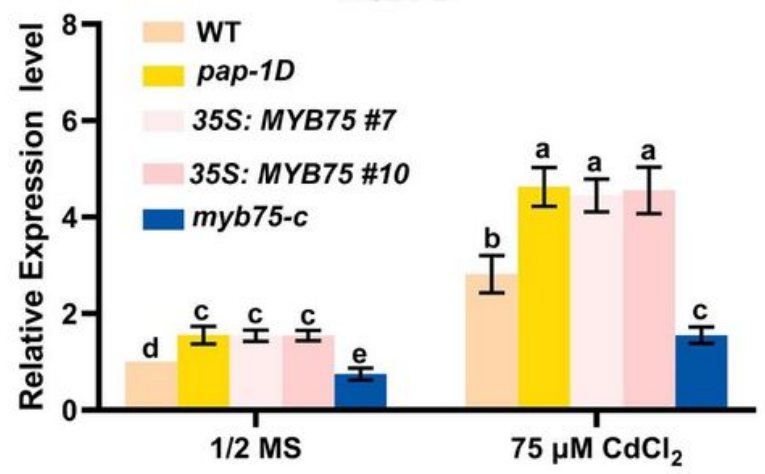

B

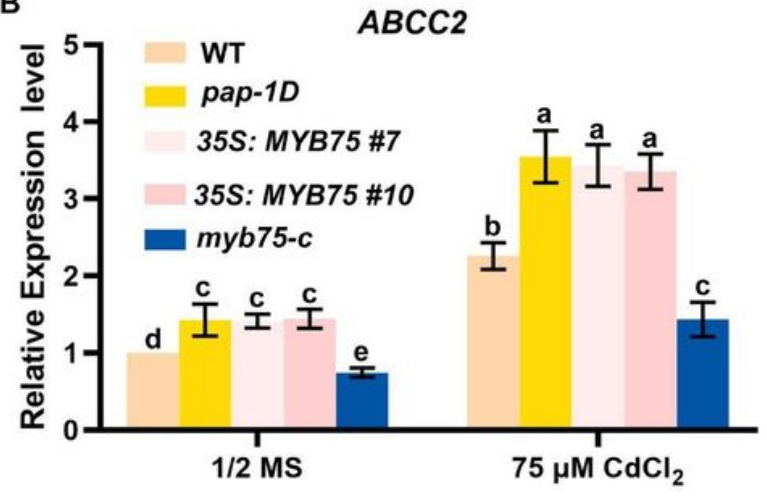

C

Reporter

//PrOACBP2 - GUS - NOS ter-/ D

I/-ProA

$35 \mathrm{~S}$

Internal Control

35S $F$

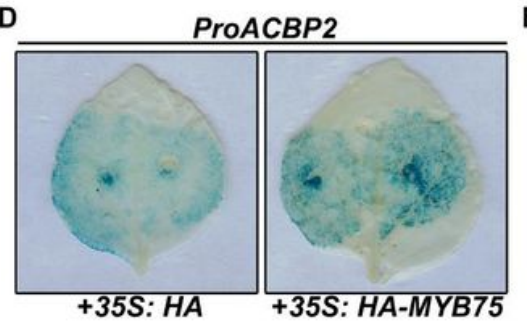

E ProABCC2

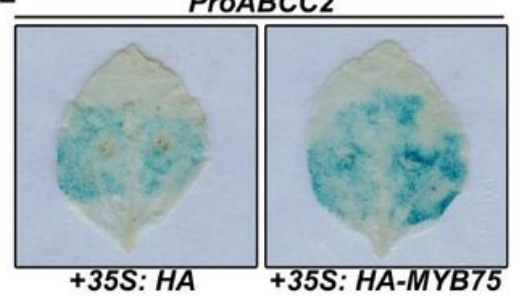

F Reporter

/I $35 \mathrm{~S}$

+35S: HA-MYB75

+35S: HA

+35S: HA-MYB75

$1 / 35 S$

G

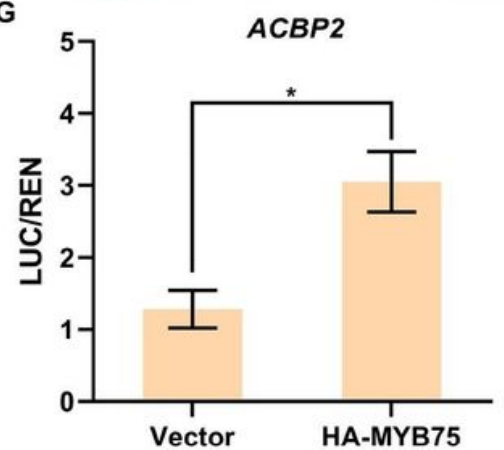

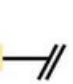

Effector

35S 35S: WYBT5-HA

$35 \mathrm{~S}$

H

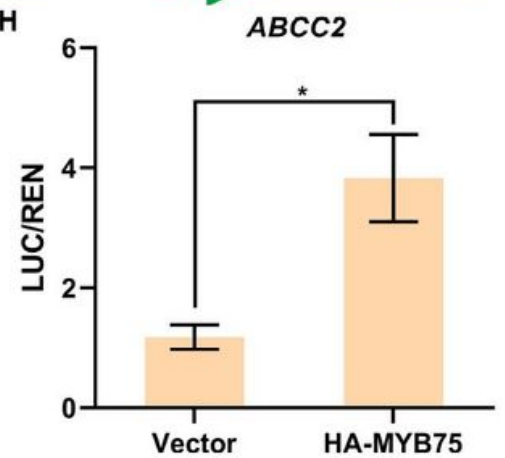

Figure 7

MYB75 regulates $A C B P 2$ and $A B C C 2$ expression. $(\mathrm{A}, \mathrm{B}) \mathrm{qPCR}$ analysis of $A C B P 2$ and $A B C C 2$ expression levels. Seedlings grown on plates for 2 weeks respectively, then treated with $75 \mu \mathrm{M} \mathrm{CdCl}_{2}$ for $4 \mathrm{~h}$, and then their mRNAs were isolated for qPCR analysis. Expression levels were standardized to ACTIN 8, the results of WT under control conditions were set at 1. Error bars denote $\pm S D(n=3)$. Different letters represented statistically significant differences (two-way ANOVA, $p<0.05$ ). (C) A schematic of the ACBP2-promoter and $A B C C 2$-promoter reporter construct, the effector plasmid, and the transfection control plasmid. (D, E) 
GUS staining of the ProACBP2: GUS and ProABCC2: GUS reporter after coexpression of 35S: MYB75-HA in Nicotiana benthamiana. Coexpression of the ProACBP2: GUS and ProABCC2: GUS reporter and 35S empty vector was used as the effector plasmid control. (F) A schematic of the ACBP2-promoter and $A B C C 2$-promoter reporter construct, the effector plasmid for LUC assays. $(\mathrm{G}, \mathrm{H}) \mathrm{LUC}$ assays of the PrOACBP2: $L U C$ and ProABCC2: LUC reporter after coexpression of 35S: MYB75-HA in Nicotiana benthamiana. Coexpression of the ProACBP2: $\angle U C$ and PrOABCC2: $L U C$ reporter and 355 empty vector was used as the effector plasmid control. Error bars denote $\pm S D(n=3)$. Asterisk represented statistically significant differences (t-test, $p<0.05)$.

A
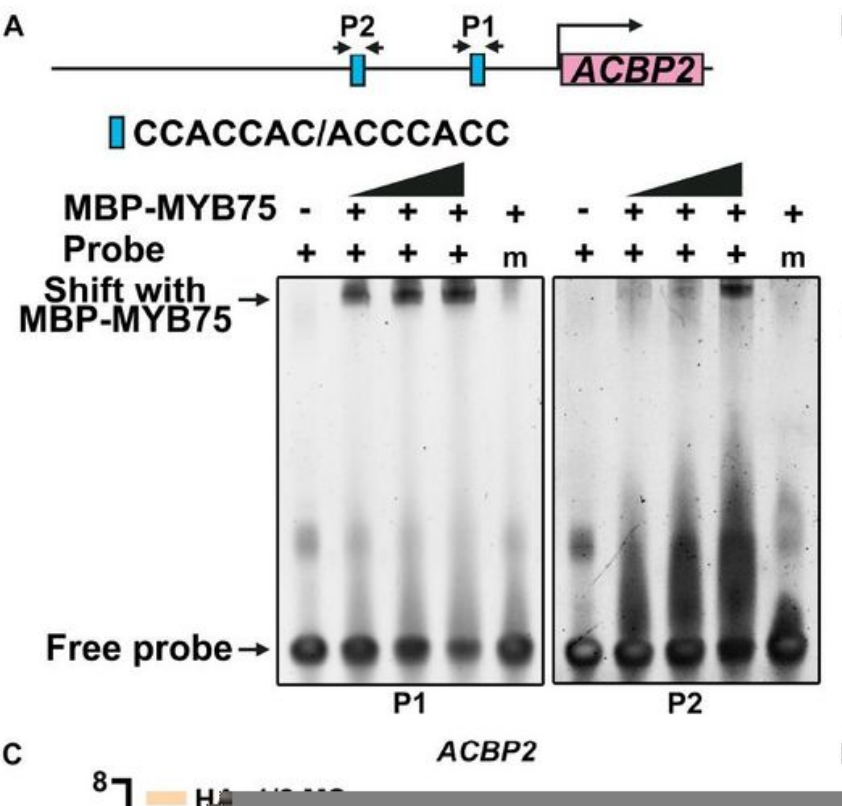

B
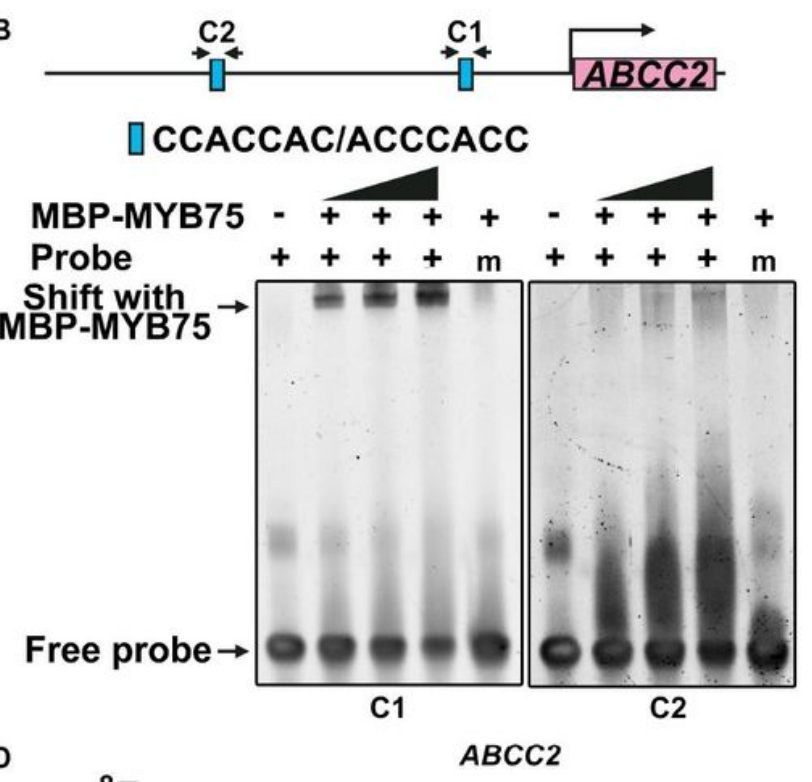

\section{Figure 8}

MYB75 directly binds to the promoter of $A B C P 2$ and $A B C C 2$. (A, B) EMSA assays of MYB75 binding to $A C B P 2$ and $A B C C 2$ promoter. Wild-type and mutant probes were incubated with MBP-MYB75, and free and bound DNAs were separated on an acrylamide gel. (C, D) ChIP assays of MYB75 binding to ACBP2 and $A B C C 2$ promoter. The 14-day-old seedlings were treated with or without $75 \mu \mathrm{M} \mathrm{CdCl}_{2}$ for $4 \mathrm{~h}$ 
respectively, then harvested for ChIP-qPCR assay using anti-HA antibody. TA3 was used as the internal control. Different letters represented statistically significant differences (two-way ANOVA, $p<0.05$ ).

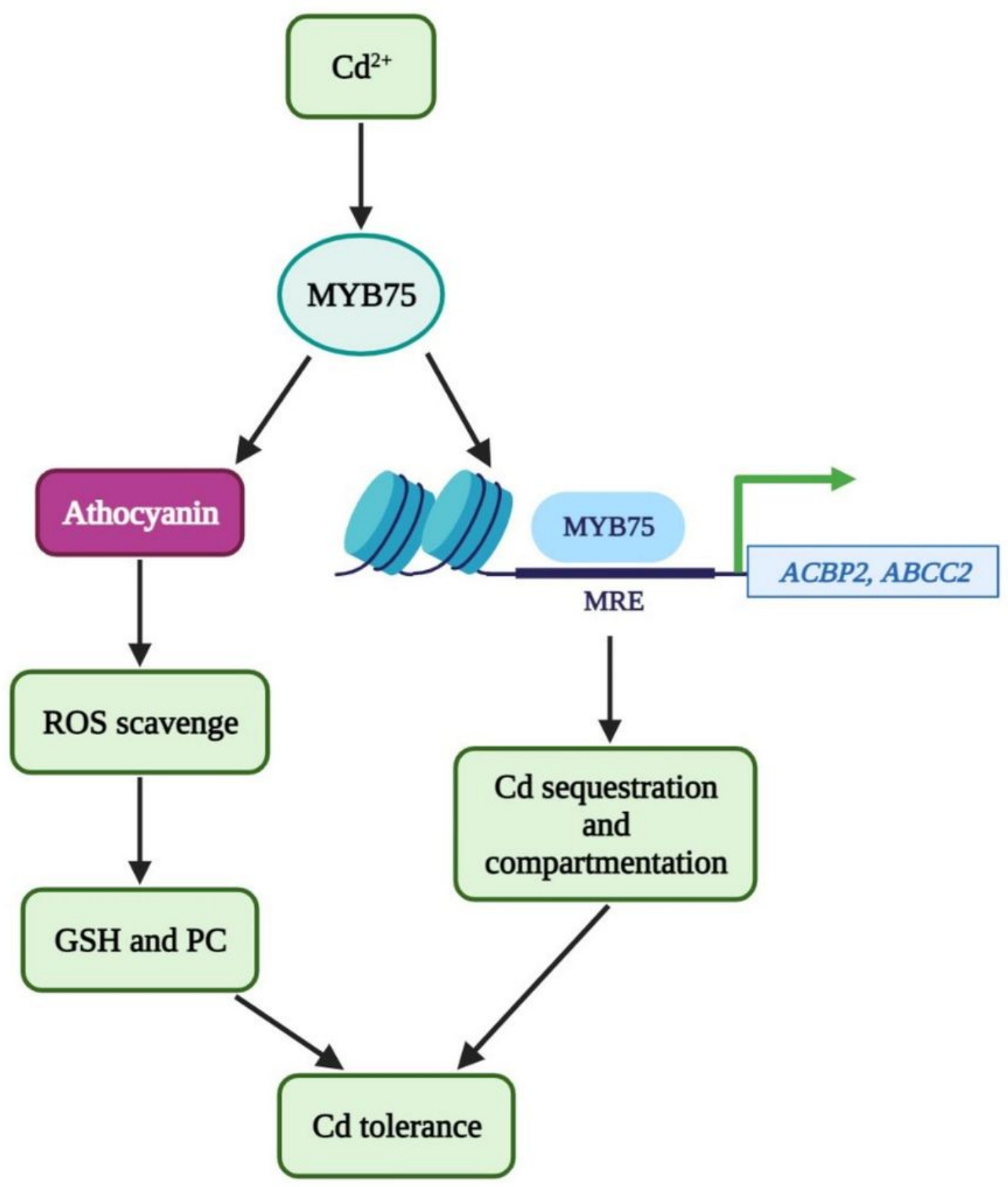

Figure 9

A working model for MYB75-regulated plant Cd tolerance. When plants were confronted with Cd stress, MYB75 transcription factor could be induced quickly. On the one hand, MYB75 activated anthocyanin 
accumulation, followed by the ROS scavenge, which resulted in the elevation of GSH and PC content. On the other hand, MYB75 directly bind to the promoter of Cd tolerance-related gene, such as ACBP2 and $A B C C 2$, thus activating their expression, which brought about $C d$ sequestration and compartmentation. The synergetic modulation via MYB75 enhanced plant tolerance in response to $\mathrm{Cd}$ stress.

\section{Supplementary Files}

This is a list of supplementary files associated with this preprint. Click to download.

- SupplementalFigure.pdf

- SupplementalTable1.docx 